\title{
Percepciones de estudiantes y docentes sobre las acciones institucionales de prevención de la violencia escolar en colegios públicos de Cúcuta
}

\author{
María de los Santos Rincón-Ramírez ${ }^{\text {a* }}$ \\ ${ }^{a}$ Magister en Prácticas pedagógicas. Docente Ministerio de Educación Nacional, \\ Secretaría de Educación Municipal de Cúcuta.
}

Forma de citar: Rincón, M. (2017). Percepciones de estudiantes y docentes sobre las acciones institucionales de prevención de la violencia escolar en colegios públicos de Cúcuta.

Perspectivas, 2(2). 32-48.

Recibido: diciembre 20 de 2016

Aceptado: abril 10 de 2017

\section{Palabras clave \\ Violencia escolar, prevención de la violencia, programas institucionales, agresión física y verbal}

Resumen: La violencia en el ámbito escolar, tanto por sus efectos inmediatos como por las repercusiones que pueda tener a futuro, es una problemática que ha preocupado a amplios sectores de la sociedad. En muchas instituciones no se reconoce su existencia y se ignoran las acciones que deberían ejecutarse para atajarla; en otras, en cambio, se enfrenta mediante acciones preventivas y correctivas que permitan menguar las dificultades de convivencia. En este contexto, el presente artículo informa sobre los resultados de un estudio sobre las percepciones de las manifestaciones de la violencia en el ámbito escolar de estudiantes y docentes de las instituciones públicas de la ciudad de Cúcuta, Colombia, y sobre las acciones institucionales para su prevención. La investigación, de tipo cuantitativa con enfoque descriptivo y carácter exploratorio, contó con una muestra estudiantil de 348 escolares de sexto a undécimo grado cuyas edades oscilan entre los 10 y 16 años; en el estudio también participaron 87 docentes de secundaria. Los resultados destacan las formas de agresión frecuentes que se presentan en las diferentes instituciones, al igual que las estrategias preventivas y las acciones para tratar casos de violencia.

\footnotetext{
*Autor para correspondencia

santicos1183@hotmail.com
} 


\section{Keywords}

School violence, prevention of violence, institutional programs, physical and verbal aggression

\section{Palavras chave}

Violência escolar, prevenção de violência, programas institucionais, agressão física e verbal

\section{Perceptions of students and teachers about institutional actions to prevent school violence in public schools in Cúcuta}

\begin{abstract}
Violence in schools is a problem that has worried broad sectors of society, not only because of its immediate effects but also due to the repercussions it may have in the future. In many institutions the violence is not acknowledged, and no actions are taken to prevent it. In contrast other institutions confront the violence head on through preventive and corrective actions that uncomplicated daily coexistence. It is in this context that this paper relays the results of a study on student and teacher attitudes to violence manifestations in the schools of Cucuta, Colombia; and on institutional prevention strategies. The quantitative-type research, executed with a descriptive and exploratory approach, used a sample of 348 students from sixth to eleventh grade, their ranging in age from 10 to 16 years old; and 87 high school teachers. The results highlight the frequently occurring forms of aggression, as well as the preventive strategies and actions schools emplace to handle cases of violence.
\end{abstract}

\section{Percepções de estudantes e professores sobre ações institucionais para prevenir a violência escolar nas escolas públicas de Cúcuta}

Resumo: A violência no ambiente escolar, tanto por causa de seus efeitos imediatos quanto pelas repercussões que pode ter no futuro, é um problema que preocupou grandes setores da sociedade. Em muitas instituições, a sua existência não é reconhecida e as ações que devem ser realizadas para detê-la são ignoradas; em outros, por outro lado, é confrontado com ações preventivas e corretivas que reduzem as dificuldades de convivência. Neste contexto, este artigo relata os resultados de um estudo sobre as percepções das manifestações de violência no ambiente escolar de estudantes e professores de instituições públicas na cidade de Cúcuta, na Colômbia, e nas ações institucionais para seus prevenção A pesquisa, de tipo quantitativo com abordagem descritiva e caráter exploratório, teve uma amostra estudantil de 348 escolares do sexto ao décimo primeiro ano, cujas idades variam de 10 a 16 anos; 87 professores do ensino secundário também participaram do estudo. Os resultados destacam as formas freqüentes de agressão que ocorrem em diferentes instituições, bem como estratégias preventivas e ações para lidar com casos de violência. 


\section{Introducción}

La violencia es un fenómeno que se percibe claramente y con marcada frecuencia en distintas esferas de la convivencia humana, ala que no escapa el ámbito escolar. Con frecuencia, en efecto, la convivencia escolar genera situaciones de liderazgo, cambios fisiológicos, control del grupo y relaciones interpersonales que producen como desenlace rivalidades y tensiones entre los estudiantes que desembocan en acciones violentas.

$\mathrm{La}$ violencia escolar tiene diferentes connotaciones. Di Leo (2008: 18), por ejemplo, señala que "En nuestra sociedad esa categoría se encuentra asociada a comportamientos o palabras inaceptables, insoportables, contrarios a la civilización, la humanidad, la modernidad".

La naturaleza de estas acciones es variada y ha sido abordada por la comunidad científica desde hace varias décadas. Entre los investigadores que ha logrado una concepción más acabada de este fenómeno se encuentran Buss y Perry (1992).

La presente investigación se aborda desde la problemática de la convivencia en las diferentes instituciones del país. Concretamente, se propone realizarun diagnóstico sobrelaviolencia en contextos escolares en colegios públicos del Municipio de Cúcuta e identificar las manifestaciones de agresión, las estrategias de prevención y las formas de atención de los casos de violencia. En este sentido, este estudio constituye un aporte para la apertura de espacios en las instituciones educativas para enseñar a mediar, escuchar y cooperar a la hora de afrontar las dificultades de violencia escolar que se presentan a diario y ofrecer espacios para la reflexión sobre la necesidad de procurar territorios de paz y convivencia en las instituciones educativas con la posibilidad de un aprendizaje emocional que facilite a los estudiantes la sana convivencia y el trabajo con los demás.

Para una mejor aproximación a la comprensión del fenómeno es necesaria la revisión teórica sobre las formas en las que se presenta la violencia en la escuela, así como el estudio de los programas de prevención y atención que se han implementado en los últimos años en las instituciones educativas.

Con relación a la prevención de la violencia en la escuela, autores como Ortega (2006), Díaz (2005), Chaux (2012), Monjas y Avilés (2006) describen programas que permiten desarrollar competencias sociales y personales para evitar la agresión y el conflicto y aumentar la empatía. En este mismo sentido, autores como MagendzoKolstrein, Toledo Jofrey Rosenfield Sekulovic(2004) aportan orientaciones preventivas que consideran claves para evitar y mediar en este tipo de conflicto. Asimismo proponen un conjunto de actividades destinadas a los profesores y padres de familia en cuento constituyen actores fundamentales para la educación en la convivencia y la paz.

Entre referentes conceptuales sobre las diferentes formas de violencia en el contexto escolar se puede citar a Valadez (2008), quien señala que las agresiones entre pares se manifiestan de forma física (daño corporal), verbal (humillar, ofender, insultar, poner motes) o psicológica (perjuicio sobre la autoestima). Dichas formas de violencia son clasificadas, de acuerdo con su intencionalidad, como de tipo ocasional o de tipo frecuente, subcategorías apoyadas en Dato (2007, citado por Castillo, 2011: 418) del modo que sigue:

El criterio para diferenciar entre violencia y acoso parece estar únicamente en la opción de respuesta dada por el estudiante. Cuando señala alguna vez, se interpreta como maltrato, como violencia; mientras si elige con frecuencia se categoriza como acoso o Bullying.

Las peleas entre iguales no siempre se ajustan a la definición del Bullying, pues no deben suponer necesariamente una agresión repetida y malintencionada para producir daño en otro escolar, al que percibe más débil. Por el contrario, estas peleas suelen ser la respuesta a conflictos de intereses o también a provocaciones recibidas. En este caso, la respuesta agresiva, bien sea física o verbal, supone el empleo de estrategias dirigidas al logro del objetivo, que representa un interés en 
relación con la resolución del conflicto. (Ortega, 2000; Smith, 2003, citado en Valadez, 2008).

Chaux (2012) expone tres manifestaciones de violencia escolar: las agresiones escolares (acciones deliberadas puntuales que buscan hacer daño a otros), los conflictos entre estudiantes (diferencias que podrían desembocar en violencia) y la intimidación (llamada hostigamiento o acoso escolar, que se produce de manera reiterada y sistemática cuando hay diferencia de fuerzas). En general, la violencia ocurre sobre los más débiles o tímidos.

En cuanto a la atención de casos de violencia en los establecimientos educativos, destacan los aportes de Martínez González y Álvarez Blanco(2005), quienes expresan que la mediación transforma los climas de convivencia social y genera instancias para el aprendizaje. Asimismo se encuentran en la literatura especializada variados programas curriculares que favorecen las relaciones de convivencia a partir de contenidos y actividades que forjan ambientes sanos. Algunas de estas propuestas y proyectos están fundamentados en las ideas de Beane(2006) y Chaux (2012).

El presente estudio busca abordar la problemática de la violencia escolar, pues pretende evaluar los distintos puntos de vista de los principales actores que forman parte de las manifestaciones de este fenómeno en el ámbito escolar: docentes y estudiantes. En concreto, se busca describir las principales percepciones que tienen estos sujetos con respecto a la existencia de acciones que puedan catalogarse como violentas y sobre las actividades y mecanismos que se establecen a nivel institucional para prevenir estos actos. El estudio se proyectó tomando como referente jóvenes con edad escolar entre 10 y 16 años que asisten como alumnos regulares a colegios públicos de la ciudad de Cúcuta, Colombia, lo mismo que los docentes de secundaria que laboran en estas instituciones.

\section{Materiales y métodos}

El estudio se fundamentó sobre una metodología de tipo cuantitativo con un enfoque descriptivo y de carácter exploratorio. A partir de una selección de instituciones educativas oficiales se abordó la evaluación de la presencia de acciones que puedan calificarse como violentas en el ámbito escolar. Para la recolección de los datos necesarios se eligió el cuestionario sobre conductas agresivas tipificadas de Buss y Perry (1992). En conjunto, se tuvo presente un enfoque del diseño en función del desarrollo y aplicación de estrategias y acciones preventivas bajo la iniciativa institucional.

\subsection{Población y muestra}

Para la investigación se escogió una muestra de 348 estudiantes de los grados sexto a undécimo, con edades comprendidas entre los 10 y los 16 años, pertenecientes a los siguientes establecimientos educativos: de carácter mixto: Eustorgio Colmenares Baptista y Carlos Toledo Plata; integrados: Juan Atalaya y Manuel Fernández de Novoa.

Los alumnos de la muestra se distribuyen de acuerdo con el género de la manera que sigue: $50,6 \%$ son del género femenino y $49,4 \%$ masculino.

En el estudio también participaron 87 docentes de secundaria, el $43,68 \%$ son mujeres y el $56,32 \%$ son hombres.

\subsection{Instrumento}

Para la recolección de datos fue necesario el diseño de un instrumento. De acuerdo con los propósitos de la investigación se seleccionó la encuesta, que estuvo basada en el cuestionario de Buss y Perry (1992) y cuyas preguntas fueron adaptadas al contexto de los participantes del estudio. Se formularon preguntas con escala Likert y preguntas multirrespuestas que, en conjunto, buscaron medir la percepción de estudiantes y docentes sobre las formas de violencia en la escuela, así como los escenarios y los medios de agresión. De igual forma indagó por medio del instrumento en las estrategias de prevención y atención de las instituciones para casos de violencia. También se incluyeron variables del perfil socio demográfico 
tales como colegio, género, edad y grado. Una vez elaborado el instrumento (tanto en su versión para estudiantes como en la destinada a los docentes) se precedió a validarlo mediante el coeficiente de Cronbach, para lo cual se aplicó a una prueba piloto de 17 estudiantes de instituciones públicas de secundaria y a 25 docentes, Los resultados de esta validación fueron de 0.7662 para el primer grupo y de 0,7725 para el segundo. Validado el instrumento, se procedió a su aplicación a la muestra de estudiantes y docentes.

\subsection{Procesamiento y análisis}

Para la sistematización de los datos se utilizó el programa Dyane (Diseño y análisis de encuestas) y la hoja de cálculo de Excel. Para la interpretación de los resultados se tuvieron en cuenta las agrupaciones de las preguntas en Existencia de violencia, Programas de prevención y Acciones institucionales para tratar casos de violencia, así como las variables colegio, grado, edad y género.

El software Dyane permitió establecer tabulaciones simples y cruzadas de las preguntas de escala Likert y multirespuesta. La hoja de cálculo Excel facilitó el cálculo de las medias aritméticas, la desviación estándar, la prueba “ $t$ ”, que permitieron evaluar las similitudes y disimilitudes de las percepciones de los encuestados sobre el fenómeno de la violencia escolar, así como evidenciar el acuerdo o desacuerdo entre ellos. Para ahondar más en las percepciones fue necesario confrontar los resultados de cada grupo encuestado, esto es, estudiantes y docentes.

\section{Resultados y discusión}

La figura 1 muestra las percepciones de estudiantes y profesores sobre la presencia de violencia escolar en las instituciones. Los valores de 3,55 y 3,66 corresponden a las puntuaciones medias de la escala Likert para estudiantes y docentes respectivamente. Estos datos revelan las declaraciones de acuerdo relacionadas con la existencia de acciones que manifiestan violencia escolar. Sin embargo, las opiniones son muy variadas, lo cual se evidencia en las desviaciones estándar. En efecto, valores superiores a 1,0 ponen de manifiesto el desacuerdo de opinión entre los encuestados. No obstante lo dicho, ni estudiantes ni profesores niegan la existencia del fenómeno.

Figura 1

Percepciones sobre la presencia de violencia escolar en las instituciones

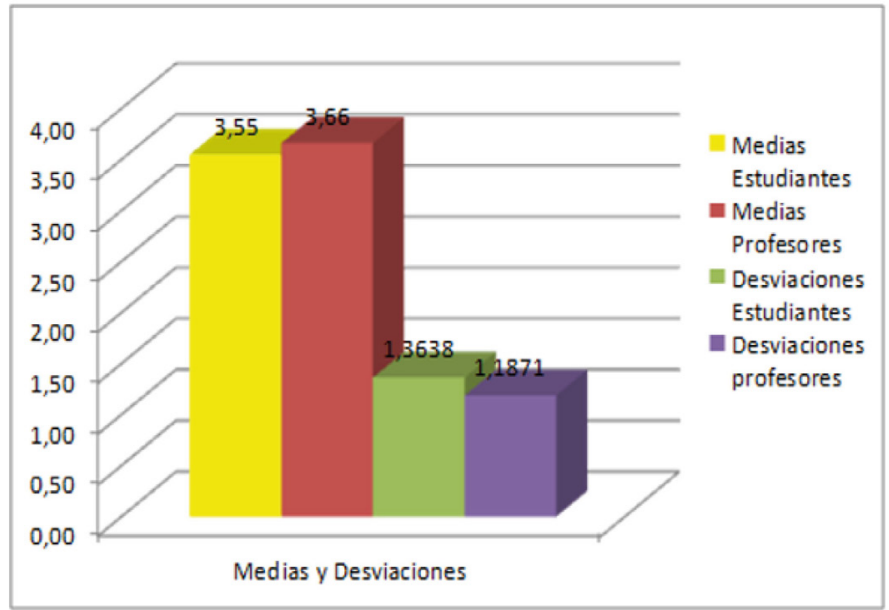

Fuente: Autor, Proceso de investigación 
Con respecto a las distintas formas en las se expresa el fenómeno, la figura 2 muestra la distribución de los quintiles tomando como indicadores acciones tales como ridiculizaciones, ofensas, amenazas, agresión física y golpes. Según la gráfica, al menos un $40 \%$ de los estudiantes expresa haber vivido episodios de amenazas o golpes en el ámbito escolar y más del $80 \%$ ha experimentado ocasionalmente situaciones de agresión verbal o física.

Figura 2

Manifestaciones de la violencia escolar

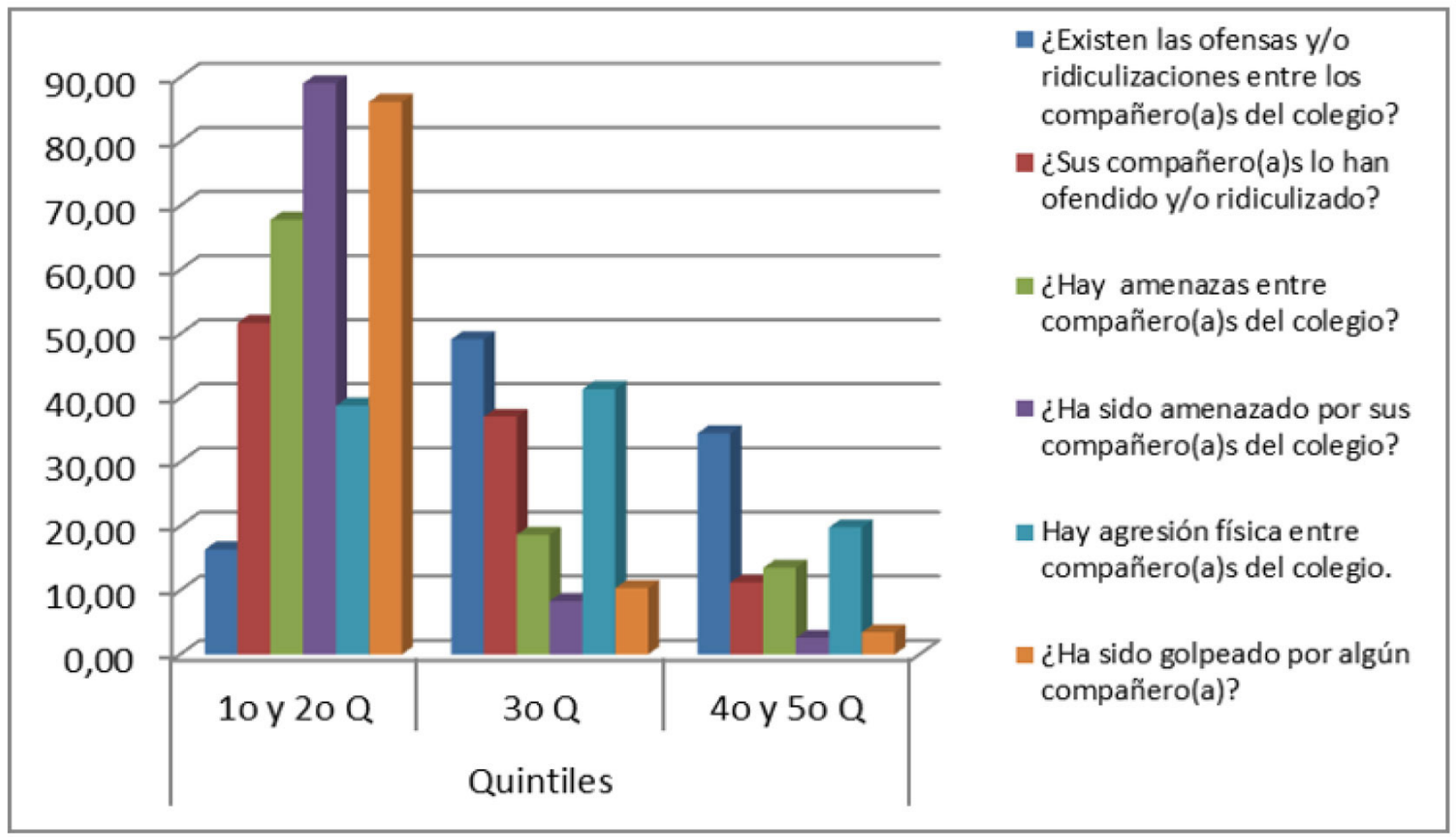

Fuente: Autor, Proceso de investigación

La figura 3 muestra las distintas acciones que lleva a cabo la institución con el fin de prevenir de la violencia escolar. Aunque al menos un $80 \%$ de los estudiantes reconoce que la institución hace esfuerzos para emprender acciones preventivas por medio de la concientización a los estudiantes, la participación es baja. En efecto, apenas un $20 \%$ del grupo ha participado en charlas y actividades relacionadas con la sensibilización hacia el tema. 
Figura 3

Valoración de las acciones institucionales y estrategias de prevención

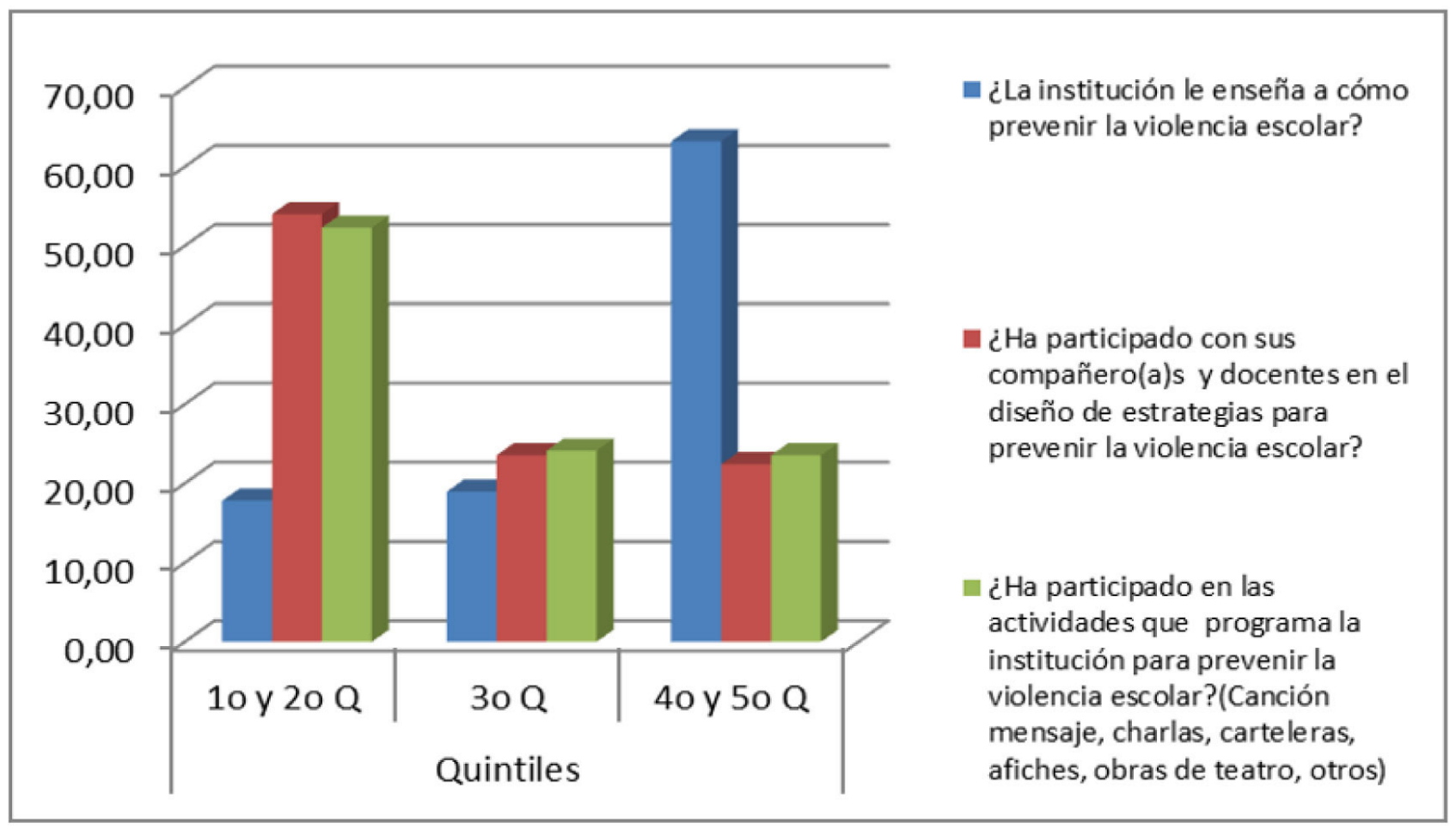

Fuente: Autor, Proceso de investigación

La figura 4 refleja lavaloración de los estudiantes de las acciones institucionales de prevención de la violencia. Como se observa, la gran mayoría de los estudiantes valoran positivamente la gestión de las instituciones educativas para promover la sana convivencia entre los escolares. Al menos el 80\% expresa la divulgación de información por medios tradicionales como carteleras y afiches, la promoción de campañas para evitar el trato discriminativo y la intervención oportuna ante situaciones de violencia. Con respecto a las denuncias, los porcentajes son más conservadores y reportan una tasa moderada aproximada al 50\% de los casos. Sin embargo, en una menor proporción (aproximadamente 20\%) los estudiantes manifiestan una visión con tendencia negativa hacia esta labor institucional. 
Figura 4

Acciones institucionales para la promoción de la sana convivencia

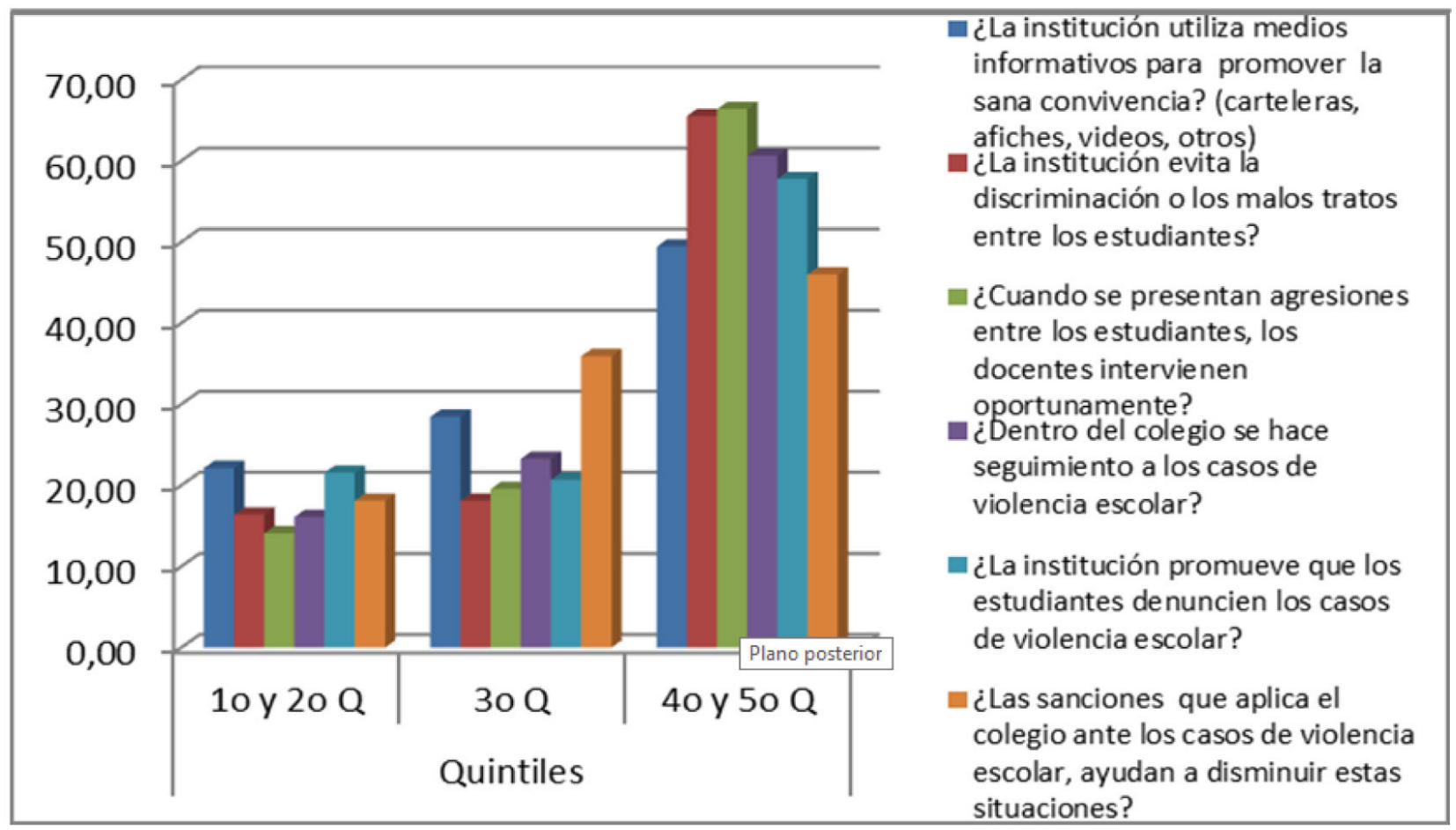

Fuente: Autor, Proceso de investigación

Considerando las variables relacionados con el perfil sociodemográfico del encuestado (colegio, género, edad y grado), se encontró, tal y como refleja la figura 5,que la comunidad de estudiantes del Colegio Eustorgio Colmenares Baptista es la que más percibe el fenómeno de la violencia, seguido del Colegio Manuel Fernández de Novoa, con una visión menos negativa sobre la presencia de este fenómeno a nivel institucional. 
Figura 5

Percepción de violencia según colegio

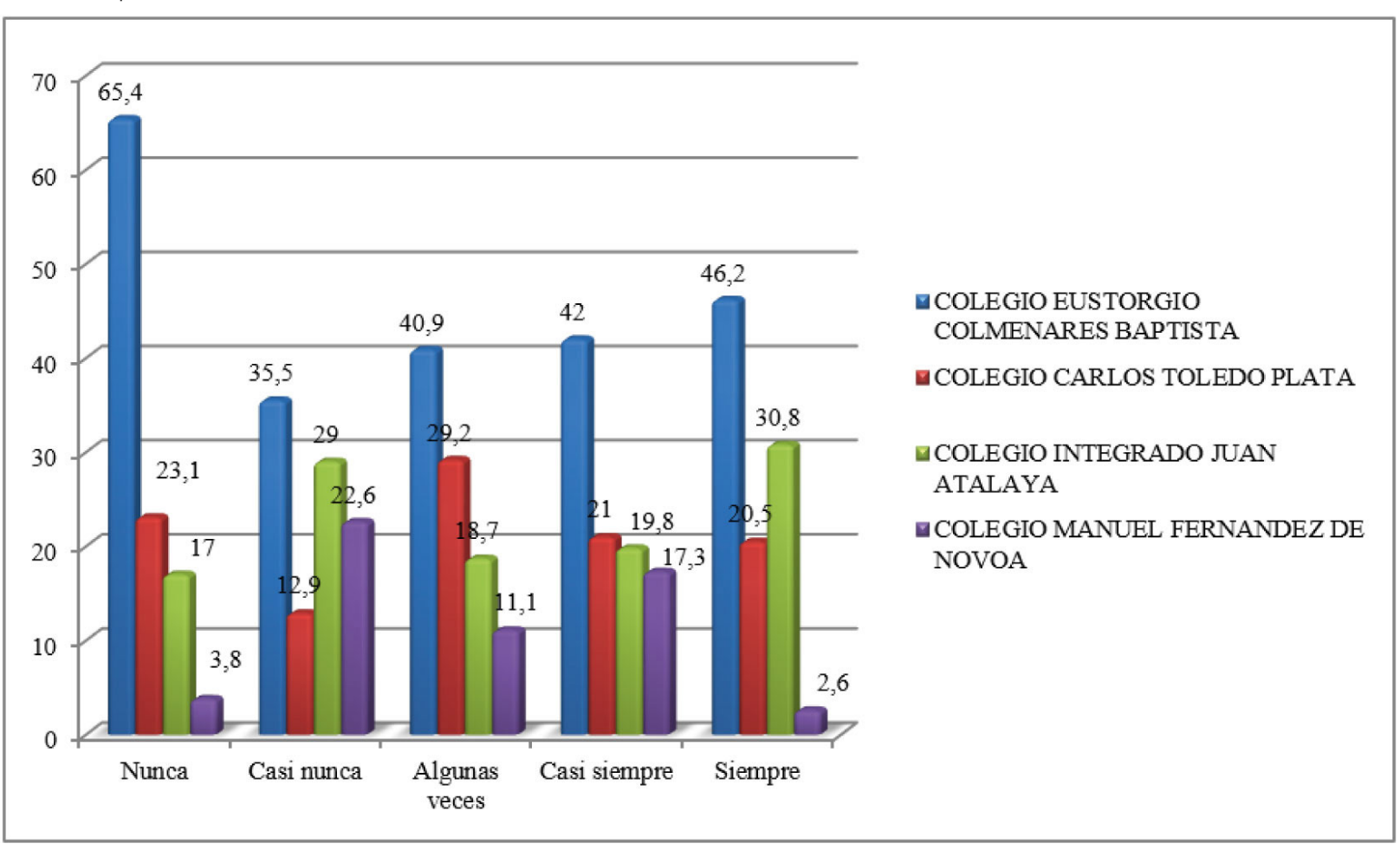

Del mismo modo se observó que, aunque no existen diferencias proporcionalmente significativas, son los hombres quienes más perciben hechos de violencia. En relación con la edad, quienes más perciben violencia en la escuela son los alumnos que están entre 13 y 15 años, seguido por el grupo cuyo rango de edad se encuentra entre los 10 y 12 años. Tal y como se evidencia en la figura 6 , el nivel escolar que más percibe la violencia es séptimo. 
Figura 6

Percepción de violencia según grado

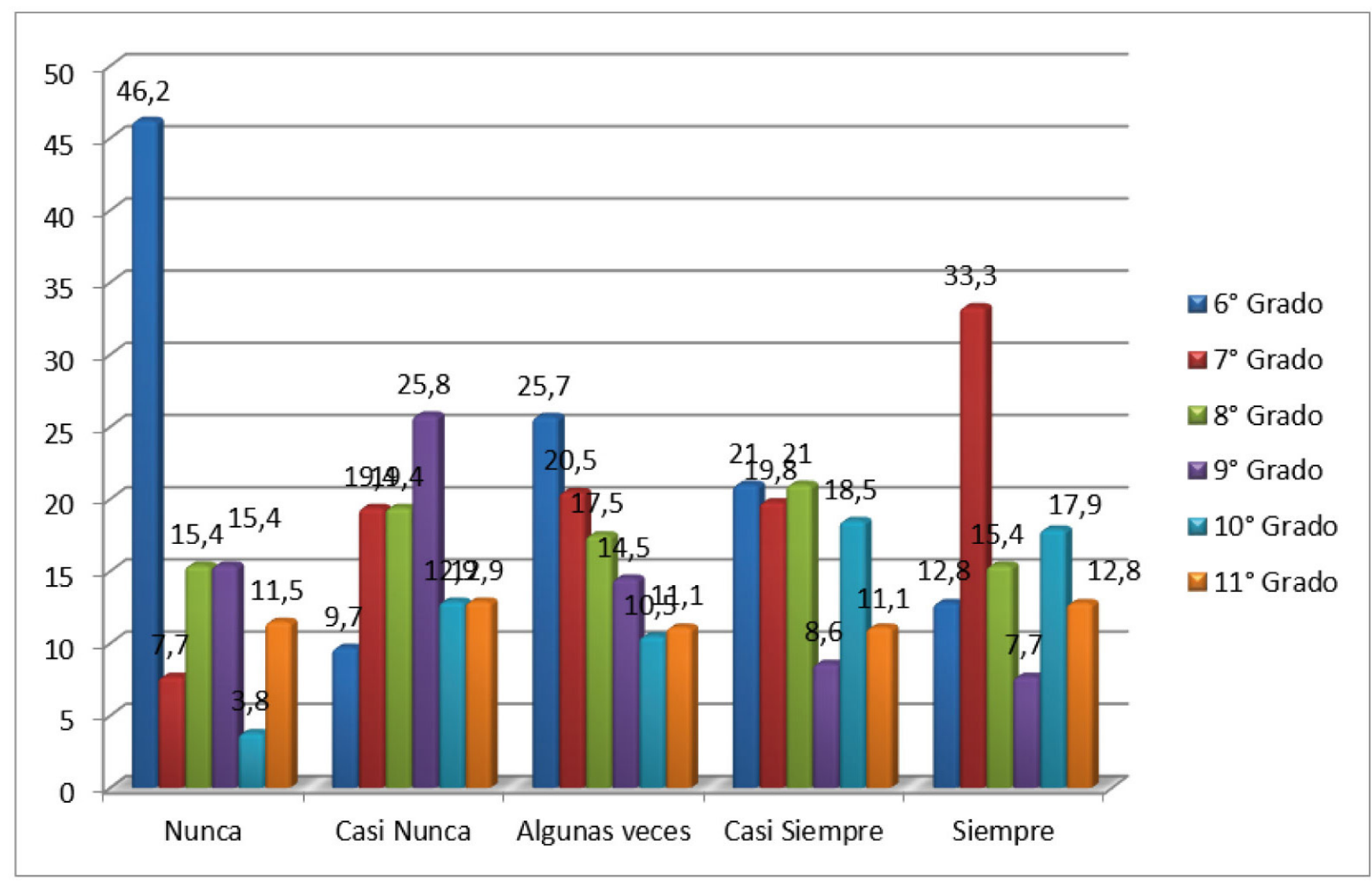

Fuente: Autor, Proceso de investigación

Un análisis comparativo de las respuestas dadas por estudiantes y docentes permite establecer los siguientes hallazgos:

a) Tanto docentes como estudiantes coinciden en que la salida del colegio es el lugar de la institución donde más se presentan hechos de violencia (28,00\% para los primeros y un $34,23 \%$ para los segundos). Igual posición ocupa el patio/cancha para los maestros $(28,00 \%)$. El salón de clase ocupa el segundo lugar para ambos grupos (27,5 para los profesores y 26,38\% para los alumnos). Otra opción destacada entre los escolares fue el patio/cancha $(21,62 \%)$. Es relevante señalar que todos los ítems fueron seleccionados en el grupo de estudiantes; no ocurrió así entre los docentes, quienes no tuvieron en cuenta la opción ningún lugar.

b) Tanto docentes como alumnos convienen en manifestar que ninguno de los medios expuestos en la encuesta son utilizados por los estudiantes para agredir a los profesores $(86,52 \%$ de los maestros y el $71,24 \%$ de los escolares). Sin embargo, otro porcentaje, aunque con puntajes menores, señalaron en segundo lugar la opción en un papel( $4,49 \%$ para el primer grupo y $15,05 \%$ para el segundo). Aparecen también para los docentes las redes sociales $(4,49 \%)$ y el tercer lugar para ambos grupos 
lo ocupa el correo electrónico. Esta vez, todos los ítems fueron considerados por los encuestados.

c) Los apodos ocupan el primer lugar para ambos grupos de encuestados en relación con las formas en que los estudiantes agreden al profesor $(38,97 \%$ de los docentes y $44,56 \%$ de los estudiantes). El segundo lugar está ocupado en la opinión de los maestros por las ofensas verbales en $(25,00 \%)$, mientras que para los alumnos ésta posición la ocupa la opción ninguna(18,89\%). Esta opción ha sido seleccionada en tercer lugar por los docentes $(15,44 \%)$, mientras para los alumnos la tercera plaza la ocupan las ofensas verbales $(12,73 \%)$.

d) Los resultados revelan diferencias de opiniones entre estudiantes y maestros, puesto que la mayoría de los docentes niegan la existencia de la discriminación en la institución $(31,15 \%)$. Por su parte, los alumnos perciben como causa de discriminación frecuente el color de la piel $(20,52 \%)$. Los maestros eligen como segunda opción la presentación personal $(22,95 \%)$ pero entre los estudiantes aparece en este lugar la apariencia física (17,03\%). En el tercer lugar de la opinión de los profesores aparece el color de piel $(14,75 \%)$ mientras que para los escolares, se trata de la presentación personal con el $(15,14 \%)$.

\section{Discusión}

A lo largo del estudio se encontró la presencia latente del fenómeno de violencia escolar en cada una de las instituciones participantes, puesto que la duda e indecisión fueron los patrones demarcadores en el proceso investigativo. Sobre este particulra, Sierra (2009) señala que

Los estudios internacionales, nacionales y locales revelan que la violencia escolar representa una realidad que se oculta y se ignora por los adultos y las instituciones, pero que está presente en casi todos los ambientes donde se generan encuentros entre personas. Fenómeno visible o invisible que aparece en forma de desprecios, chantajes, robos, peleas, discriminación, diferencias, humillación, segregación, silencio y exclusión.(Sierra, 2009, p.82).

En relación con el nivel de escolaridad, se halló que el grado que más percibe y es víctima de maltrato es el séptimo. Estos resultados coinciden con los de Hoyos, Aparicio y Córdoba (2005), quienes encontraron que el maltrato entre iguales se concentra en los grados sexto y séptimo; en especial en séptimo. Otra investigación que concuerda con estos datos es la de Cepeda, Pacheco, García y Piraquive(2008), que encontraron que las situaciones de agresión son más frecuentes en los grados inferiores: sexto, séptimo y octavo.

En razón de las edades, la investigación encontró que los estudiantes que se ubican en los rangos de 13 a 15 años son quienes con mayor frecuencia sufren agresiones. En este sentido Sweeney (2008), citado por Mendoza (2011) hace referencia a que en sus investigaciones las edades con mayor incidencia de maltrato escolar se ubican en los rangos de los 13 a los 15 años, y comienza a disminuir a los 16 años. Así mismo Díaz (1999), citado por Revollo, Medrano, Olier y Acosta (2008), caracteriza este período como de rebeldía del proceso madurativo y es cuando los conflictos se resuelven en su mayoría de forma violenta.

En cuanto al género, el estudio corroboró que los escolares hombres son quienes mayormente perciben y son víctimas de agresiones, tantos verbales como físicas. En esto coincide Díaz (2005), los resultados de cuya investigación revelaron que los varones manifiestan un riesgo superior de violencia y de intolerancia que las mujeres, De igual forma Cangas, Gázquez, Pérez, Padilla y Miras (2007) encuentran que son los hombres quienes se sienten más afectados por las agresiones. Revollo, Medrano, Olier y Acosta (2008) hallaron igualmente que entre estudiantes de básica secundaria la agresividad fue más usual en los varones que en las mujeres. Tappery 
Boulton (2004), citados por Benítez y Justicia (2006), señalan que son los hombres los que se ven implicados con mayor frecuencia en situaciones de violencia escolar.

En cuanto a los tipos de agresión más comunes entre escolares, docentes y estudiantes determinaron que son las ofensas verbales, seguidas de la agresión física. Mendoza (2011) manifiesta que el abuso verbal es la forma más común de violencia en las escuelas, que consiste en utilizar palabras malintencionadas hacia un niño o joven minando su bienestar moral, físico o mental. Hoyos, Aparicio y Córdoba (2005) concluyen en su investigación que la manifestación que caracteriza el maltrato entre iguales es el maltrato verbal. De igual forma Oliveros, Figueroa, Mayorga, Cano, Quispe y Barrientos (2008), que estudiaron los tipos de agresión más frecuentes entre estudiantes, encontraron que la verbal fue mucho más usual, que la física. También Postigo, González, Mateu, Ferrero y Martorell (2009) concluyen que las agresiones verbales se dan reiteradamente y que las agresiones físicas son minoritarias. Un estudio cuyos resultados van en dirección contraria es el de Revollo, Medrano, Olier y Acosta (2008), quienes destacan que la agresión física es la más concurrente en los escolares.

Con respecto a la participación en peleas, la mayoría de los escolares señalaron no haber intervenido en ellas de ninguna forma, hecho que corrobora que el fenómeno de la agresión física se presenta en las instituciones estudiadas en escasa proporción. Una minoría de los estudiantes, sin embrago, señaló haber participado como observadores, o también defendiéndose o separando a los actores. En este sentido, Trianes (2000) citado por Prieto, Carrillo y Jiménez (2005) encuentra que los espectadores se caracterizan por no participar en peleas directamente, pero están presente en la riñas o son observadores del acoso. En relación con la anterior, Mendoza (2011) caracteriza a los espectadores como "los secuaces" (amigos que acompañan al agresor), "los alentadores" (que son quienes estimulan las reacciones agresivas en los demás), "los ajenos o indiferentes" (que permanecen neutrales y con indiferencia) y "los defensores" (que intervienen abandonando el papel de espectador, apoyan a la víctima, denuncia el acoso y condenan o desaprueban al agresor). En cuanto a la participación en peleas para defenderse, podría inferirse que esta situación se ocasiona en respuesta a una agresión inicial y que la que desencadena más actos violentos. Chaux (2012) es de esta opinión, y señala que al responder una agresión con otra agresión se alimenta la violencia. Prieto, Carrillo y Jiménez (2005) concluyen en su estudio que los alumnos son simultáneamente víctimas y agresores en términos de respuesta a conductas violentas recibidas. El sentirse un alumno lastimado o herido constantemente lo lleva a defenderse y posiblemente a convertirse en agresor (Mendoza, 2011).

Entre los escolares encuestados existen percepciones distintas con respecto a la existencia de violencia por parte de los docentes hacia los estudiantes. Un alto porcentaje, en efecto, señala que no existen actos violentos por parte de los profesores; sin embargo, una minoría señala que sí se presentan en formas de ridiculización, insultos y golpes. Valdivieso (2009) pone de manifiesto mediante su investigación que ridiculizar al estudiante es una de las formas frecuentes de agresión de los docentes. Asimismo, de acuerdo con los resultados de su trabajo, tanto estudiantes como maestros coinciden en manifestar que los conflictos entre profesores y escolares se ocasionan por los comportamientos inadecuados de un grupo de alumnos. En este sentido Gómez (2005) indica que los episodios de agresión de los docentes hacia el estudiante se originan como consecuencia de conductas inapropiadas del escolar, que no incluyen a la totalidad del alumnado, y como forma de ratificar las jerarquías de poder. Chaux (2002), por su parte, encontró en un estudio colombiano que el $15 \%$ de los estudiantes reportaron haber sido víctimas de insultos por un docente en la última semana.

Desde el punto de vista de maestros y escolares se encontró que las formas más frecuentes en que los estudiantes agreden al profesor son los apodos y las ofensas verbales. Prieto (2005) argumenta 
que para los estudiantes es común ponerle apodos a los docentes, probablemente porque en su entorno ellos también son objeto de motes. Arellano (2004) plantea que existen manifestaciones de violencia escolar hacia los docentes cuya manifestación consiste en ofensas verbales, agresiones físicas, deterioro de sus bienes y materiales. Sobre esta particular, Chaux (2012) señala que los docentes también son víctimas de maltrato e intimidación, ya sea por estudiantes, directivos o padres de familia.

En la investigación se halló igualmente que la mayoría, tanto de docentes como de escolares, conviene en que no han sido agredidos a través de ninguno de los medios expuestos en la encuesta. No obstante, en ambos grupos se encuentran porcentajes menores que ha señalado que esta agresión se manifiesta mediante escritos en un papel, las redes sociales y el correo electrónico. Estas dos últimas se consideran nuevas modalidades de acoso y van en aumento. De acuerdo con Mendoza (2011: 39 ), "se refiere a la violencia y ataques ejercidos por medios electrónicos, ya sea a través de mensajes de textos en los celulares, en los chats, o en los blogs". En el estudio realizado por Jouvon en en la Universidad de California (UCLA) citado por Mendoza (2011) se encontró que tres de cada cuatro adolescentes habían sido agredidos en línea al menos una vez en los últimos doce meses. Las agresiones virtuales van en rápido crecimiento (Chaux2012).

Los dos grupos encuestados coinciden en señalar la salida del colegio como el espacio de la institución donde con mayor frecuencia se presentan hechos de violencia. También destacan el salón de clase y el patio/cancha. Estos datos son similares a los de Muñoz, Saavedra y Villalta (2007), quienes encontraron que la salida del colegio es momento y el lugar en el que ocurren las peleas entre pares. Por su parte, Valdivieso (2009) pone de manifiesto que el lugar de deporte y el salón de clase son lugares de la escuela donde se presentan episodios de agresión. Hoyos, Aparicio y Córdoba (2005) también encuentran que el patio es el lugar con mayor potencial de aparición de acciones violentas.
Los resultados también revelan ciertas las diferencias de opiniones en torno al tema de la violencia. La mayoría de los docentes, por ejemplo, niega la existencia de discriminación en la institución; sin embargo otro grupo de maestros señalan como acto discriminatorio la presentación personal y el color de piel. Los alumnos, por su parte, perciben como discriminación frecuente el color de piel, la apariencia física y la presentación personal. En concordancia con estos resultados, Valdivieso (2009) señala que los ámbitos discriminatorios más recurrentes están basados en las características físicas, discapacidades físicas y mentales y de particularidades de la personalidad.

En cuanto a la programación de estrategias para prevenir la violencia escolar, los resultados ponen en manifiesto la duda que se encuentra en los estudiantes de los colegios Eustorgio Colmenares Baptista, Carlos Toledo Plata y Manuel Fernández de Novoa, así como la tendencia a la negación de dichas estrategias en el colegio Integrado Juan Atalaya. Entre los docentes de los diferentes colegios, por otra parte, se percibe la indecisión en razón de las mencionadas estrategias, lo que confirma la duda existente entre los grupos encuestados. En efecto, al confrontar las percepciones de escolares y maestros se encontró que estos difieren en sus opiniones. El desacuerdo no solo se encuentra entre las instituciones sino también según el género, los rangos de edad y los grados. En este orden de ideas se podría considerar que las actividades preventivas se hacen de forma esporádica y que la participación de la comunidad educativa en cuanto al diseño y ejecución de la misma es limitada. De acuerdo con este hallazgo es fundamental resaltar la importancia de realizar acciones continuas que favorezcan la convivencia pacífica entre los miembros de una institución. Así lo sugiere Díaz (2005) cuando señala que los programas de prevención resultan más eficaces cuando se desarrollan de manera continua y a largo plazo. El autor también plantea que para mejorar la convivencia y prevenir la violencia escolar es necesario intervenir lo antes posible, sin esperar las graves manifestaciones que suelen alertar 
sobre dicha necesidad. Mendoza (2011) indica que una de las razones importantes para prevenir la violencia es la magnitud con la que crece. Si no se frenan los comportamientos violentos, estos tienden a propagarse, pues se favorece la impunidad. Por otra parte, la violencia genera violencia y si ésta no se atiende a tiempo llega a potenciarse hasta el punto de perderse el control sobre la misma. Mendoza (2011) también afirma que para resolver cualquier problema primero hay que reconocerlo e incluir en las acciones a todos los miembros de la comunidad educativa, pues lo expertos consideran que se trata de la única vía posible de prevención de la violencia en el ámbito escolar.

Con respecto a las acciones institucionales para atender casos de violencia, docentes y estudiantes del colegio Manuel Fernández de Novoa coinciden en manifestar que la labor institucional es efectiva. La percepción de los encuestados del colegio Carlos Toledo Plata tiende a ser favorable, mientras en el Eustorgio Colmenares Baptista e Integrado Juan Atalaya predomina la duda con respecto a la pertinencia de estas acciones. Cabe resaltar que tanto estudiantes como profesores concuerdan en mostrar como una de las acciones relevantes "la intervención oportuna de los docentes en los casos de agresión", hecho que podría hacer suponer que los episodios de violencia en las diferentes instituciones están siendo controlados por maestros y directivos y por ende poco se visibiliza el fenómeno. Valdivieso (2009) concluye en su estudio que los profesores que intervienen en las conductas de maltrato tienden a estar más alertas y son menos condescendientes con los maltratadores, Así también, Hoyos, Aparicio y Córdoba (2005) dedujeron en su estudio que la mayoría de los profesores intervienen para detener la situación de maltrato. Sin embrago, la investigación de Prieto (2005) mostró que los maestros conocían de las acciones violentas de los estudiantes y no hacía nada por evitarlo. Por su parte Chaux (2012) señala que cuando no se atienden de manera oportuna y adecuada las agresiones, se gesta violencia. En este sentido Monclús (2005) afirma que la intervención es un proceso participativo, que no es suficiente transformar el comportamiento del agresor, sino también modificar las conductas de los espectadores. Esta idea va en línea con Mendoza (2011), quien indica que para detener el fenómeno de la violencia escolar es necesario vincular a todos los miembros de la institución, hecho que se evidencia en el estudio al encontrar que la institución integra a la familia para tratar casos de violencia, así como también promueve la denuncia de los casos de agresión, Díaz (2005) respalda acciones de este tipo, pues es de la opinión de que se debe enseñar al alumno a decir no y pedir ayuda en situaciones de abuso.

\section{Conclusiones}

A lo largo del estudio se pudo apreciar que la violencia escolar no solo se concibe en las comunidades educativas como maltrato reiterado, acoso u hostigamiento. En efecto, no solo se interpreta ni se mide por la cantidad de episodios de agresión que se producen contra un individuo. Sencillamente la violencia es y está en la escuela. Se ve reflejada en el diario vivir, en peleas entre los escolares, sin que exista necesariamente desigualdad de fuerza o poder. Las agresiones no tienen que ser reiterativas para que sean tenidas en cuenta o sean consideradas como distractor de la convivencia pacífica. De acuerdo con muchos expertos, la violencia está presente en todas las instituciones educativas y no depende del nivel socioeconómico; sus diversas manifestaciones no consideran género ni jerarquías de poder. Alertan, además, sobre el hecho de que las situaciones violentas más van tomado forma y apoderándose cada vez de las aulas y otros espacios pensados para la socialización del individuo. Si estas situaciones no se erradican puede ocasionar daño no solo a los directamente involucrados sino también a los que se posicionan alrededor como espectadores pasivos o activos.

En suma, "la violencia escolar es una realidad incuestionable que tiene múltiples formas y se da en varias direcciones: de autoridades a alumnos y maestros, de maestros a alumnos y de alumnos a alumnos" (Prieto, Carrillo, Jiménez 2005:3) 
En el presente estudio se alcanzó a observar que no existen diferencias significativas de opinión entre estudiantes y docentes en cuanto a la percepción del fenómeno de la violencia escolar, programas de prevención y acciones institucionales para tratar casos de agresión.

De igual forma en los encuestados predominó la duda con respecto a la existencia de la violencia en las instituciones, pues en el marco de sus respuestas, mientras algunos niegan hechos violentos, otros, aunque en minoría, perciben episodios de agresión. Ello permite sugerir que el fenómeno de la violencia está presente en todas las instituciones educativas, sin que sea necesariamente reconocido abiertamente por sus miembros.

En cuanto a las manifestaciones de violencia en las instituciones se encontró que son más frecuentes las agresiones verbales que la física. Por otra parte, los hombres son quienes más perciben y son víctimas de agresión. Con respecto al nivel de escolaridad, el grado séptimo está más expuesto a episodios de maltrato, así como también los estudiantes con edades comprendidas entre los 13 y los 15 años. Los tipos de discriminación frecuentes son debidas al color de la piel, presentación personal y apariencia física.

Los resultados demostraron que aunque un alto porcentaje de estudiantes niega la participación en peleas; otro revela que su intervención ha sido como espectador, en función de defensa o separando a los involucrados directos. Todo lo anterior constituye comprobaciones de que los escolares están involucrados con frecuencia en hechos de agresión. Así también, un grupo de escolares devela que los profesores agreden al estudiante en forma de ridiculización, insultos y golpes. De modo similar, los estudiantes agreden al docente al ponerle apodos y ofenderlos verbalmente. En proporción semejante, un porcentaje de alumnos y otro de profesores ponen de manifiesto que han sido ofendidos a través de papeles, redes sociales y correo electrónico.

Se halló igualmente que existe muy poca participación de estudiantes y docentes en el diseño y ejecución de estrategias para prevenir la violencia escolar. Pero es preciso reconocer que los resultados revelaron que las instituciones aplican acciones para tratar casos de violencia y que estas en su mayoría resultan efectivas. Asimismo se encontró que es "la intervención de los docentes en situaciones de violencia" la acción más destacada, con lo cual se podría considerar que la minimización de la violencia encontrada en el estudio es producto de una labor eficaz por parte de los maestros y los directivos. Acciones a tiempo pueden contrarrestar los hechos de agresiones actuales y en la misma medida prevenir futuras situaciones de maltrato.

En conclusión, el fenómeno de la violencia requiere de atención minuciosa, tanto para prevenirlo como, en el caso de que se manifieste y tratarlo a tiempo. Considerando que la escuela es el lugar donde los individuos construyen la idea de sociedad y es allí donde se consolidan los valores aprendidos en el hogar y donde se hacen evidentes las diferentes manifestaciones de solidaridad, cooperación, así como de autoridad y dominio propio, es de vital importancia desde la escuela diseñar y aplicar estrategias que favorezcan la convivencia pacífica entre los escolares, así como crear pautas para combatir a tiempo el fenómeno de la violencia escolar, llevar a cabo campañas que expongan la magnitud del problema, las consecuencias y formas de prevenirlo.

\section{Referencias}

Arellano, N. (2004). Comunicación en la Prevención del conflicto en instituciones educativas de Media, Diversificada y Profesional. Tesis doctoral no Publicada. Maracaibo: Universidad Rafael Belloso Chacín.

Beane, A. (2006). Bullying. Aulas libres de acoso. Barcelona: Graó.

Benítez, J. y Justicia, F. (2006). El maltrato entre iguales: Descripción y análisis del fenómeno. Revista electrónica de investigación psicoeducativa, (4), 9: 151-170.

Buss, A.H. y Perry, M.P. (1992). The aggression questionnaire. Journal ofPersonality and Social 
Psychology, 63: 452-459.

Cangas, A.; Gázquez, J.; Pérez, M.; Padilla, D. y Miras, F. (2007). Evaluación de la violencia escolar y su afectación personal en una muestra de estudiantes europeos. Psicothema, 19(1):114119.

Castillo, L. (2011). El acoso escolar. De las causas, origen y manifestaciones a la pregunta por el sentido que le otorgan los actores.Magis. Revista Internacional de Investigación en Educación, 4 (8): 415-428.

Cepeda, E., Pacheco, P., García, L. yPiraquive C. (2008). Acoso escolar a estudiantes de educación básica y media. Salud Pública, 10 (4): 517-528.

Chaux, E. (2002). Buscando pistas para prevenir la violencia urbana en Colombia: conflictos y agresión entre niños (as) y adolescentes en Bogotá. Estudios Sociales, 12: 43-53

Chaux, E. (2012). Educación, convivencia y agresión escolar. Bogotá: Prisa.

Di Leo, P.(2008). Violenciasyescuelas:Desplieguedelproblema. EnKornblit, A. (Coord.).Violencia escolar y climas sociales. Buenos Aires: Biblos, págs. 1741.

Díaz, A. (2005). Por qué se produce la violencia escolar y cómo prevenirla. Revista Iberoamericana de Educación, 37: 17-47.

Gómez, A. (2005). Violencia e institución educativa. Revista mexicana de investigación educativa, 10 (26): 693-718.

Hoyos, O.; Aparicio, J. y Córdoba, P. (2005). Caracterización del maltrato entre iguales en una muestra de colegios de Barranquilla, Colombia. Psicología desde el Caribe, 16: 1-28.

Magendzo Kolstrein, A.; Toledo Jofre, M.J. y RosenfieldSekulovic, C. (2004). Intimidación entre estudiantes. Cómo identificarlos y cómo atenderlos. Santiago de Chile: LOM.

Martínez González, R. y Álvarez Blanco, L. (2005). Fracaso y abandono escolar en Educación Secundaria Obligatoria: implicación de la familia y los centros escolares. Aula Abierta, 85: 127-146.
Mendoza, M. (2011). La violencia en la escuela: Bullies y víctimas. México D.F.: Trillas.

Monclús, A. (2005). La violencia escolar: Perspectivas desde Naciones Unidas. Revista Iberoamericana de Educación, 38: 1332.

MonjasyAvilés(2006).Programa de sensibilización contra el maltrato entre iguales.Valladolid: Consejería de Educación. Junta de Castilla y León.

Muñoz, M. Saavedra, G. Villalta, M. (2007). Percepciones y significados sobre la convivencia escolar de estudiantes de cuarto medio de un liceo municipal de Chile. Revista de Pedagogía, 28(82): 197-224.

Oliveros, M., Figueroa, L., Mayorga, G., Cano, G., Quispe, Y. y Barrientos, A. (2009). Intimidación en colegios estatales de secundaria del Perú. Revista Peruana de Pediatría, 62: 68-78.

Postigo, S.; González, R.; Mateu, C.; Ferrero, J. y Martorell, C. (2009)Diferencias conductuales según género en convivencia escolar. Psicotema, 21(3): 453-458.

Prieto, M. (2005). Violencia escolar y vida cotidiana en la escuela secundaria. Revista mexicana de investigación educativa, 10(27): 1005-1026.

Prieto, M.; Carrillo, J. y Jiménez, J. (2005). La violencia escolar: Un estudio en el nivel medio superior. Revista mexicana de investigación educativa, 10(27): 1027- 1045.

Revollo, A.; Medrado, Y.; Olier, E. y Acosta, A. (2008). Caracterización de comportamientos agresivos en estudiantes de básica secundaria, de las instituciones educativas La Unión y Nueva Esperanza de la zona sur de Sincelejo. Revista de Investigación y Pedagogía, 1(1): 9-23.

Sierra, C. (2009). Manifestaciones de violencia en la escuela primaria: elementos de perfilación de víctimas y agresores. Panorama, 7: 98-116.

Valadez, I. (2008). Violencia escolar: maltrato entre iguales en escuelas de secundaria de la zona metropolitana de Guadalajara. México.

Valdivieso, P. (2009). Violencia escolar y relaciones intergrupales. Sus prácticas y significados 
en las escuelas secundarias públicas de la comuna de Peñalol en Santiago de Chile. Tesis Doctoral Inédita. Granada: Universidad de Granada. Disponible en http://hera.ugr.es/ tesisugr/18070206.pdf 